\section{Occurrence of thermotolerant Campylobacter in raw poultry meat, environmental and pigeon stools collected in open-air markets}

\author{
Alberto Bellio, ${ }^{1}$ Amaranta Traversa, ${ }^{1}$ \\ Daniela Adriano, ${ }^{1}$ \\ Daniela Manila Bianchi,' \\ Alberto Colzani,'2 Stefano Gili, ${ }^{2}$ \\ Alessandro Dondo, ${ }^{1}$ Silvia Gallina, ${ }^{1}$ \\ Carla Grattarola, ${ }^{1}$ Cristiana Maurella, ${ }^{1}$ \\ Simona Zoppi, ${ }^{1}$ Fabio Zuccon, ${ }^{1}$ \\ Lucia Decastelli ${ }^{1}$
}

1'Istituto Zooprofilattico Sperimentale del Piemonte, Liguria e Valle d'Aosta, Torino; ${ }^{2}$ Azienda Sanitaria Locale TO1, Torino, Italy

\section{Abstract}

Campylobacteriosis was the most commonly reported zoonosis for confirmed human cases in European Union during 2011. Poultry meat was very often implicated in Campylobacter infections in humans. In Italy commerce of raw poultry meat is common in open-air markets: these areas can be considered at high risk of bacterial contamination due to the high presence birds like pigeons. The aim of this study was to collect data about the contamination by thermotolerant Campylobacter of raw poultry meat commercialised in open-air markets, of work-surfaces in contact with poultry meat and of pigeon stools sampled in the market areas in Turin, Northern Italy. Between September 2011 and December 2012, 86 raw poultry meat samples, 86 environmental swabs and 108 animal samples were collected in 38 open-air markets. Analysis were carried out according to IS010272-1:2006 standard. C.coli was detected in $2.3 \%(2 / 86)$ of raw poultry meat samples, whereas no swab $(0 / 86)$ resulted positive. Of pigeon stool 28\% (30/107) was positive for C.jejuni (83.3\% C.jejuni subsp. jejuni and $16.7 \%$ C.jejuni subsp. doylei). C.jejuni subsp. jejuni was isolated from 1 dead pigeon. Our results showed lower rates of contamination than those reported at retail in Europe. Although samples were collected in areas at high risk of contamination, raw poultry meat and work surfaces reported a low level of presence of thermotolerant Campylobacter. The high percentage of $C$.jejuni isolated from pigeon stools showed the importance of a continuous application of preventive measures by the food business operators and the surveillance activity by the Competent Authority.

\section{Introduction}

Thermophilic Campylobacter, especially Campylobacter jejuni and Campylobacter coli, are considered the most common bacterial agent of human gastroenteritis in developed countries. Directive 2003/99/EC (European Commission, 2003) establishes the monitoring of zoonoses and zoonotic agent such as Campylobacter spp. This approach shall include each production step in the food chain: from farm level to the retail sale. Campylobacter jejuni and Campylobacter coli are distributed in the intestinal tract of many asymptomatic birds and mammals that are considered the main reservoirs for these microrganisms. Transmission to humans mainly occurs through consumption or handling of animal origin foods; moreover, contact with infected animals may determine human campylobacteriosis (OIE, 2008). The disease is usually characterised by acute gastrointestinal signs represented by diarrhea, abdominal pain, nausea, vomiting, fever and headache. Symptoms occur one to five days after infection and last for several days. Campylobacteriosis cases may be rarely associated to reactive arthritis, Guillain-Barré syndrome, nephritis and hepatitis. In the European Union (EU), human campylobacteriosis is increasing with a statistically significant rate of confirmed cases from 2008 to 2011 among 13 reporting Member States. In 2011 confirmed cases of human campylobacteriosis in EU were 200,209 (EFSA, 2013): this positive trend was characterised by a clear seasonal factor. Human cases were reported more frequently during summer months, when ambient temperature is higher. In fact, a study conducted in the EU showed that the season can represent an important risk factor for poultry contamination [OR=7.4, CI $(9.3 ; 5: 36)$ in the period from July to September compared with JanuaryMarch] (EFSA, 2011). In addition to summer temperatures, it must be considered that the high number of flies (from July to September) could represent a mechanical vector for contamination of poultry meat (Hald et al., 2008). Actually, raw chicken meat is considered the major source of human campylobacteriosis in Europe. Surveys conducted at retail level, often showed high contamination values by thermophilic Campylobacter: $38.4 \%$ (435/1132) in fresh meat and meat products of poultry in Switzerland (Baumgartner and Felleisen, 2011), $87.2 \%$ (321/368) in raw poultry meat samples collected at some supermarkets in Poland (Wieczorek et al., 2012), and 46.8\% $(146 / 312)$ in broiler meat products in Lithuania (Kudirkiene et al., 2013).

Open-air markets are important reference points in the urban economy, as citizens can find fresh food at low cost. Fresh meat sale at
Correspondence: Fabio Zuccon, Istituto Zooprofilattico Sperimentale del Piemonte, Liguria e Valle d'Aosta, via Bologna 148, 10154 Torino, Italy.

Tel. +39.11.2686233 - Fax: +39.11 .2473450 .

E-mail: fabio.zuccon@izsto.it

Key words: Open-air market, Thermotoleran Campylobacter, Raw poultry meat, Pigeon stools.

Conflict of interests: the authors declare no potential conflict of interests.

Received for publication: 13 May 2013

Revision received: 2 January 2014

Accepted for publication: 28 January 2014

This work is licensed under a Creative Commons Attribution 3.0 License (by-nc 3.0).

(C) Copyright A. Bellio et al., 2014

Licensee PAGEPress, Italy

Italian Journal of Food Safety 2014; 3:1706

doi:10.4081/ijfs.2014.1706

the market areas usually takes place in proper equipped automarkets, which often do not ensure the same working conditions of shops and supermarkets. In particular, the lack of water limits appropriate equipment washing. Automarkets have refrigerated counters where fresh meat is exposed: this should guarantee the correct refrigeration temperature, providing a partially physical barrier against microbiological agents.

In Turin area there are about 40 open-air markets: one of these is the largest open-air market in Europe, called Piazza della Repubblica, where many and varied foodstuffs such as fruit and vegetables, dairy products, fresh and processed meat are sold.

The aim of this study was to verify the presence of thermophilic Campylobacter in poultry meat sold in the open-air market in Turin area and simultaneously assess the level of environmental contamination of faecal origin from wild animals, such as rock pigeon.

\section{Materials and Methods}

A total of 280 samples were collected in 38 open-air markets in Turin, from September 2011 to December 2012. Samples (137 in 2011 and 143 in 2012) included poultry meat, swabs of surfaces where poultry is processed, stools of rock pigeon (Columba livia) and a dead pigeon. Meat samples and environmental swabs were collected at 86 counters in 30 market areas. In each sale counter a sample of poultry meat ( 84 chicken and 2 turkey meat samples) and a surface swab were collected. In 
particular, poultry samples can be distinguished into breast $(\mathrm{N}=23)$, thigh with skin $(\mathrm{N}=41)$, wings with skin $(\mathrm{N}=1)$, whole chicken $(\mathrm{N}=1)$ and unknown $(\mathrm{N}=20)$; environmental swabs were performed on the working plane $(\mathrm{N}=64)$, on knife blade $(\mathrm{N}=19)$ and unknown $(\mathrm{N}=3)$. A $100 \mathrm{~cm}^{2}$ of the working planes area was sampled, while on knife blades both sides of the entire surface were swabbed. Swab sampling was carried out by wiping back and forward the whole surface rapidly. The area was first swabbed with one side of the swab and then it was swapped again with the opposite side of the same swab. A total of 108 biological samples (107 stool and 1 dead pigeon) was collected in 36 market areas. Stool samples were collected in the period JanuaryMarch $(\mathrm{N}=6)$, in the second $(\mathrm{N}=18)$, third $(\mathrm{N}=32)$ and fourth quarter $(\mathrm{N}=51)$. Sampling was carried out by veterinarians of the local public health service (ASL T01). Samples were transported at refrigeration temperature to the Istituto Zooprofilattico Sperimentale Piemonte, Liguria and Valle d'Aosta (IZSPLV), where microbiological analyses were performed.

Environmental swabs and food samples were processed according to ISO 10272-1:2006 (ISO, 2006) method. Faecal samples and the dead pigeon animal were analysed according to ISO 10272-1:2006 method (OIE manual of diagnostic tests and vaccines for terrestrial animals). Identification of Campylobacter species was performed by biochemical system API Campy (API-bioMérieux SA, Marcy l'Etoile, France).

\section{Results}

Out of 280 samples, 33 (11.8\%) resulted positive for thermophilic Campylobacter. In particular, among the 86 meat samples only two (2.3\%) resulted positive for Campylobacter coli: one sample of chicken breast in 2011 and one sample of chicken thigh in 2012 , both collected in November, in two different markets of Turin area (open-air market in Piazza Vittoria and in Piazza della Repubblica). All food contact surfaces were negative. Out of 107 faecal samples, 30 (28\%) resulted positive for Campylobacter jejuni (12 samples in 2011 and 18 samples in 2012). C. jejuni subspecies jeju$n i(83.3 \%)$ was the most frequently subspecies isolated in stool samples, followed by $C$. jejuni subsp. doylei (16.7\%). C. jejuni subsp. jejuni was isolated from the dead pigeon collected in 2011 (Table 1). Stool samples collected during the January-March period showed the highest level of contamination with $100 \%(6 / 6)$ positive followed by the October-December period with $30 \%(15 / 51)$; finally the second quarter with $28 \%(5 / 18)$ and the third with $12.5 \%$ (4/32). Considering stool samples, $52.7 \%$ (19/36) of sampled areas resulted positive for
Table 1. Results of samples collected from September 2011 to December 2012.

\begin{tabular}{lcccccccc} 
& \multicolumn{3}{c}{2011} & & \multicolumn{2}{c}{2012} \\
& Meat Swabs & Stool & Dead pigeons & Meat Swabs & Stool Dead pigeons \\
C. coli & 1 & 0 & 0 & 0 & 1 & 0 & 0 & 0 \\
C. jejuni subsp.jejuni & 0 & 0 & 9 & 1 & 0 & 0 & 16 & 0 \\
\hline C. jejuni subsp. doylei & 0 & 0 & 3 & 0 & 0 & 0 & 2 & 0 \\
Negative & 41 & 42 & 40 & 0 & 43 & 44 & 37 & 0 \\
\hline Total & 42 & 42 & 52 & 1 & 44 & 44 & 55 & 0 \\
\hline
\end{tabular}

C.coli, Campylobacter coli; C.jejuni subsp. jejuni, Campylobacter jejuni subspecies jejuni; C.jejuni subsp. doylei, Campylobacter jejuni subspecies doylei.
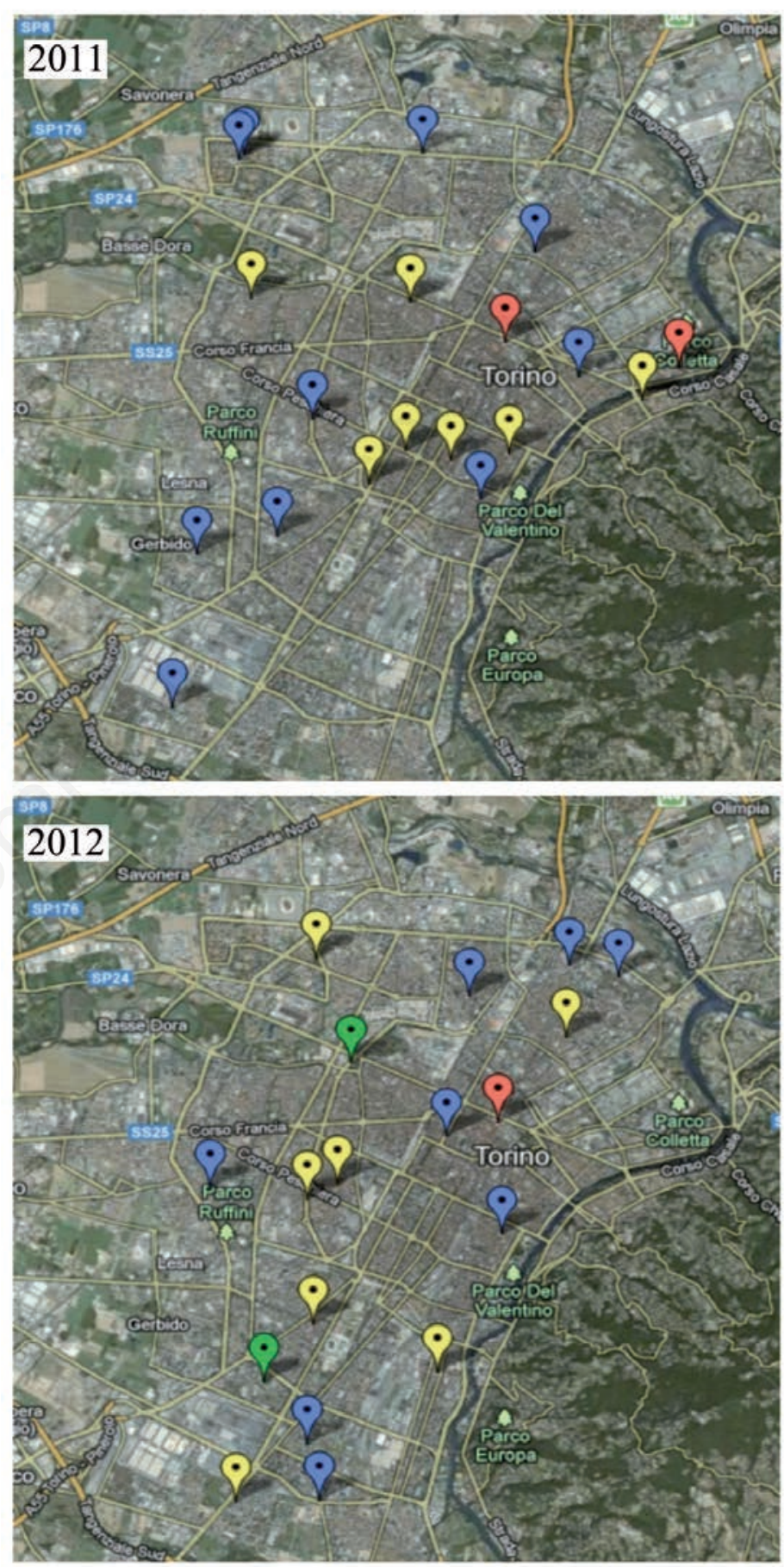

Figure 1. Market areas where stool samples were collected: negative area (blue), positive

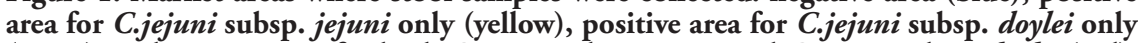

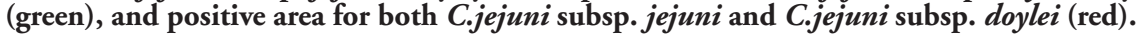


thermophilic Campylobacter. Stool samples collected from open-air market of Piazza della Repubblica showed the highest percentage of positivity: 50\% of samples (2/4) in 2011 and $90 \%(9 / 10)$ in 2012 . Market areas where stool samples were collected are showed in Figure 1.

\section{Discussion}

High concentrations of animals in areas such as public gardens and market areas, could be a risk for citizens' health: direct contact with infected animals or contaminated material thereof could represent a source of Campylobacter spp. contamination for humans (OIE, 2008).

This survey showed that $52.7 \%$ of the market areas, sampled for pigeon faecal material, resulted positive for Campylobacter spp.: the high prevalence of positive samples (28\%) demonstrates how birds could be considered as vectors and reservoirs for Campylobacter spp. in urban environment. Even if only pigeon stools were collected, other urban species as well, such as sparrows, starlings and seagulls, should be considered a possible source of contamination. Moreover, it should not be underestimated the role of insects, such as flies, which may be a mechanical means of food contamination in open-air markets (Hald et al., 2008). Prevalence of Campylobacter in poultry meat $(2.3 \%)$ confirms data obtained in Piedmont region during the national monitoring plan on broiler carcasses at slaughter (Di Giannatale et al., 2010). Over the Italian territory, however, other authors reported a higher level of contamination (40.8\%) in fresh poultry meat at retail (Prencipe et al., 2007).

C. coli was isolated from food samples only (breast and chicken leg samples), while $C$. jejuni was detected only in stool samples. Positivity of pigeon faecal material did not show a seasonal trend. Prevalence observed during the first quarter of the year (100\%) may be linked to the low sample size $(\mathrm{N}=6)$. Percentages obtained in the second (28\%) and fourth (29.4\%) quarter were similar, while during summer the prevalence was lower than the others (12.8\%). Most samples (31/32) tested in summer, were collected in September.

The analysis of geographical location of Campylobacter spp. positivity did not allow to detect any spatial aggregations: environmental contamination was distributed over the urban territory without the possibility to identify areas in which the two subspecies were isolated. Market areas are generally characterised by high concentration of people and animals, with counters structural and management limits and with a common exposure of foodstuffs to open air. For all these reasons, open air markets could be considered potential areas at high risk for food microbiological contamination.

\section{Conclusions}

The microbiological data reported in the present study show a low contamination level both in poultry meat and environmental swabs. Considering the characteristics of poultry meat as raw material and the working conditions, structural and management limits of market areas, these data demonstrate a low risk of contamination by thermophilic Campylobacter. On the contrary, the high prevalence of positive stool samples should be taken into account, because of the high density of pigeons in market areas in Turin. These birds could be considered a source of contamination for environment and food. This evidence suggests the need for correct and continuous application of preventive measures by the food business operators supported by the surveillance activities carried out by the Competent Authority.

\section{References}

Baumgartner A, Felleisen R, 2011. Market surveillance for contamination with thermotolerant Campylobacters on various categories of chicken meat in Switzerland. J Food Protect 74:2048-54.

Di Giannatale E, Prencipe V, Colangeli P, Alessiani A, Barco L, Staffolani M, Tagliabue S, Grattarola C, Cerrone A, Costa A, Pisanu M, Santucci U, Iannitto G, Migliorati G, 2010. Prevalenza di
Campylobacter termotolleranti nel pollo da ingrasso in Italia. Veter Ital Ser 46:405-14.

EFSA, 2011. Scientific opinion on Campylobacter in broiler meat production: control options and performance objectives and/or targets at different stages of the food chain. EFSA J 9:2105.

EFSA, 2013. The European Union summary Report on trends and sources of zoonoses, zoonotic agents and food-borne outbreaks in 2011. EFSA J 11:3129.

European Commission, 2003. Directive of the European Parliament and of the Council of 17 November 2003 on the monitoring of zoonoses and zoonotic agents, amending Council Decision 90/424/EEC and repealing Council Directive 92/117/EEC, 2003/99/EC. In: Official Journal, L 325/31, $12 / 12 / 13$.

Hald B, Skovgard H, Pedersen K, Bunkenborg $\mathrm{H}, 2$ 2008. Influxed insects as vectors for Campylobacter jejuni and Campylobacter coli in Danish broiler houses. Poultry Sci 87:1428-34.

ISO, 2006. Microbiology of food and animal feeding stuffs. Horizontal method for detection and enumeration of Campylobacter spp. Part 1: detection method. ISO Norm 10272-1:2006. International Standardization Organization ed., Geneva, Switzerland.

Kudirkienė E, Bunevičienė J, Sernienè L, Ramonait S, Olsen JE, Malakauskas M, 2013. Importance of the producer on retail broiler meat product contamination with Campylobacter spp. J Sci Food Agr 93:22938.

OIE, 2008. Manual of diagnostic tests and vaccines for terrestrial animals. Available from: http://www.oie.int/manual-of-diagnostic-tests-and-vaccines-for-terrestrialanimals/

Prencipe V, Parisciani G, Calistri P, Caporale CM, Iannitto G, Morelli D, Pomilio F, Prochowski D, Migliorati G, 2007. Veter Ital Ser 43:157-65.

Wieczorek K, Szewczyk R, Osek J, 2012. Prevalence, antimicrobial resistance, and molecular characterization of Campylobacter jejuni and C. coli isolated from retail raw meat in Poland. Vete MedCzech 57:293-9. 